\title{
INCIDENCIA DEL DAÑO MECÁNICO EN SEMILLAS DE POROTO (PHASEOLUS VULGARIS L.) SOBRE EL DESARROLLO Y EL RENDIMIENTO DE PLANTAS NORMALES Y ANORMALES
}

\author{
BEHAVIOUR OF MECHANICALLY DAMAGED SEEDS ON \\ THE DEVELOPMENT AND YIELD OF NORMAL AND ABNORMAL \\ BEAN (PHASEOLUS VULGARIS L.) PLANTS
}

\author{
Amalia S. Romano ${ }^{1}$; I. Teves ${ }^{1}$; L. Cazón ${ }^{1}$
}

\section{RESUMEN}

El daño mecánico es otro factor determinante del deterioro de la semilla. El objetivo del presente trabajo fue analizar su incidencia sobre el desarrollo y el rendimiento de plantas normales y anormales de dos cultivares de poroto. Para ello, sublotes de semillas de poroto blanco Paloma INTA y de poroto negro Camilo INTA fueron dañados dejándolos caer: cero, dos y cuatro veces sobre un plato metálico desde una altura de dos metros. Se valoraron porcentajes de germinación, Índice de vigor de plántulas normales y anormales, además de sus ingresos a las fases R6 (floración), R7 (formación de vainas) y R9 (madurez a cosecha), números de vainas, de semillas/planta y de semillas/vaina, longitud de vainas y peso de 1.000 semillas, calculándose finalmente un Índice de cosecha. Mientras la germinación sólo en poroto negro mostró mermas -aunque no significativas- en el porcentaje de plantas normales para el tratamiento cuatro caídas, el Índice de vigor evidenció, para las plantas anormales de todos los tratamientos de poroto blanco, declinaciones significativas respecto de las normales, comportamiento que sólo se repitió en el cultivar Camilo para el tratamiento de cuatro caídas.

En cuanto a la duración de las fases reproductivas, las plantas normales de poroto blanco provenientes de los tratamientos dos y cuatro caídas y las anormales de cuatro caídas adelantan las fases R6, R7 y R9, mientras que las correspondientes anormales de poroto negro sólo adelantaron las fases R6 y R9. Además, para este cultivar, las plantas normales de dos caídas y las anormales del lote testigo mostraron retrasos en sus ingresos a las fases R6 y R7 en comparación con sus respectivos controles.

Al analizar la productividad final se observó que -mientras en poroto blanco las plantas anormales del tratamiento dos caídas mostraron declinaciones significativas $(\mathrm{p} \leq 0,05)$ en los números de vainas y de semillas/planta- ambos componentes del rendimiento aumentaron entre un 19-37\% respectivamente en las plantas testigo de poroto negro; y en menor proporción para el tratamiento dos caídas, en cada caso en comparación con sus respectivas plantas normales.

En cuanto al Índice de cosecha, el mismo mostró incrementos de hasta un 65\% para las plantas anormales respecto de las normales de testigo y dos caídas de poroto blanco; y para el tratamiento cuatro caídas de poroto negro se observó un aumento del 13\%. Estos resultados -si bien muestran a Paloma como el más susceptible al daño mecánico- en relación a la similitud de comportamientos observados entre ambos cultivares frente al rendimiento de los tipos de plantas analizadas (normales/anormales) estarían indicando la existencia de alguna asociación de las plantas anormales con efectos de tipo compensatorio.

Palabras clave: Daño mecánico, poroto, desarrollo, rendimiento, planta normal, planta anormal.

\section{ABSTRACT}

Mechanical damage is a factor determining deterioration of seed. This research was aimed at analyzing its incidence on the development and yield of normal and abnormal plants of two bean cultivars. For this purpose, sublots of seeds of the white bean Paloma INTA and the black one Camilo INTA were damaged by letting them fall on a metallic plate: none, two, and four times, from a two meter height.

Germination percentages, vigour index of normal and abnormal seedling, as well as their arrival into the phases R6 (flowering), $R 7$ (pods formation) and $R 9$ (maturity at harvest), number of pods and seeds/plant, seeds/pod, length of pods, weight of 1,000 seeds, were assessed; finally, a harvest index was calculated.

1 Universidad Nacional de Jujuy. Facultad de Ciencias Agrarias. San Salvador de Jujuy. Jujuy. Argentina. E-mail: amaliaromano@ hotmail.com 
Germination percentage showed decreases-although not significant-only in the case of normal black bean plants with four falls; whereas the vigour index showed significant decreases as compared with normal of all white bean treatments. This was also the case for the Camilo cultivar with four falls.

Regarding the length of reproductive phases, normal plants of white bean, from the two and four falls treatments, and abnormal plants from the four falls advanced the R6, R7 and R9 phases; whereas the corresponding black bean abnormal ones advanced only the R6 and R9 phases. Besides, the normal plants of this cultivar with two falls and abnormal ones of the control lot showed delays in their arrivals to phases R6 and R7, as compared with their controls.

When the final productivity was analyzed, it was noticed that-whereas the abnormal white bean plants from the two falls treatment showed significant $(p \leq 0.05)$ decreases in the number of pods and that of seeds/plant-both components of yield increased by 19 to $37 \%$ respectively in the case of black bean control plants; and in lower proportion for the two falls treatment in each case, as compared with their respective normal plants.

Regarding the harvest index, it showed increases up to $65 \%$ for abnormal plants as compared with normal ones of the control and two falls of white bean; and for the four falls treatment of black bean a $13 \%$ increase was observed.

These results -although showing Paloma as the most sensitive one to mechanical damage, in relation to behaviour similitudes observed facing the yield of both types of plants analyzed (normal/abnormal) - would indicate the existence of some association of the abnormal plants, with compensatory effects.

Key words: Mechanical damage, bean, development, yield, normal plant, abnormal plant.

\section{INTRODUCCIÓN}

La valoración del potencial fisiológico que poseen las semillas constituye una herramienta fundamental para tomar decisiones tanto en las diferentes etapas del proceso de producción como para el almacenamiento y en la comercialización (Colete et al., 2004).

Entre los trabajos realizados con el propósito de evaluar la calidad de la semilla, la mayoría sostiene que esta característica ejerce una particular influencia sobre la producción económica de numerosos cultivos, al afectar el establecimiento y el crecimiento de las plántulas, y también su productividad (Finch-Savage, 1995).

La calidad fisiológica de un lote de semillas es la resultante de dos momentos en la historia de la planta madre: en primer lugar su habilidad para producir semillas vigorosas y tolerar la sequía y en segundo término por las declinaciones que sufre el vigor de las mismas, promovidas por mecanismos de deterioro que se suceden durante la pérdida de humedad de la semilla (Powell et al., 1984). La merma de esa calidad ocurre durante los procesos que se suceden desde su maduración en el campo hasta el almacenamiento, la que además se acentúa por efectos de altas temperaturas y humedad (Begnami \& Cortelazzo, 1996; Walters et al., 2001). Es así como la semilla inicia una serie de transformaciones degenerativas que conducen a su deterioro (Bewley, 1986; Trawatha et al., 1995).

Otro factor determinante del envejecimiento de la semilla es el daño mecánico, el que puede ser inducido de manera artificial durante la cosecha, en el acondicionamiento y tambien en el transcurso de las operaciones que se realizan para la siembra (Tekrony, 2003). En legumbres resultan más susceptibles a este deterioro las semillas grandes blancas que las que poseen tegumento de color. Sobre ello, estudios realizados por Kannenberg \& Allard (1964) les permitieron sostener que la cubierta de semillas blancas brinda menor protección al embrión que la que ofrecen aquellas de tegumentos coloreados. Así el material dañado evidencia alteraciones de membranas celulares, cambios a nivel de ácidos nucleicos y reducciones de las actividades enzimáticas, las que conducen a una germinación más lenta, mermas en la tasa de crecimiento, incrementos en el número de plántulas anormales y finalmente pérdidas en el poder germinativo, características que definen a los lotes de semillas de bajo vigor (Delouche \& Baskin, 1973).

Por otro lado, estudios comparativos del crecimiento que presentan las plántulas normales y anormales de maní aseguran que si bien durante los primeros 13 días desde la germinación las anormales evidencian menor porte que las plantas normales, a medida que avanza el ciclo del cultivo y por efectos de tipo compensatorio se observan incrementos similares en el crecimiento entre ambos tipos de plantas (Lovey, 1999).

Respecto de los días transcurridos desde la siembra hasta la aparición de la primera flor, se conocen resultados contradictorios. Así mientras Sullivan \& Perry (1976) observaron variaciones en el inicio de la floración en plantas de maní, otro estudio realizado con la misma especie (Lovey, 1999) no encontró diferencias que resultaran significativas $(\mathrm{p} \leq 0,05)$ al comparar ese comportamiento entre plantas normales y anormales. 
Si se analiza la productividad, investigaciones realizadas en soja sostienen la existencia de variabilidad en la habilidad que tiene el grano para formar plántulas normales y fuertes. Por su parte, las plantas débiles y anormales producían menor número de vainas y de granos/planta, con lo que decrecía el rendimiento de esa especie (ISTA, 1985). Sin embargo, a partir de estudios abordados por Prijic et al. (1991) se comprobó que las plantas anormales de soja son capaces de alcanzar durante la estación de crecimiento algún grado de recuperación, llegando a rendimientos que no difieren significativamente de los valorados a partir de plantas normales y vigorosas. Asimismo se verificó que el número de vainas y de granos/ planta son caracteres directamente asociados con el rendimiento en cultivos de granos.

Por otro lado, trabajar con Índices de cosecha es una manera apropiada de conocer las variaciones que se producen en los rendimientos en leguminosas, como lo demostraron en las últimas décadas las relaciones de peso seco de granos /peso seco de parte aérea calculados para varios cultivos (Araujo et al., 1999). Sobre el particular, estudios efectuados en distintos cultivares de poroto reportaron diferencias en las correlaciones calculadas entre rendimientos en granos e Índices de cosecha, resultados que posiblemente se deben a las adaptaciones genéticas logradas en sus regiones de origen. Por otra parte, se sostiene que el Índice de cosecha permite reforzar la importancia que posee la partición de la biomasa hacia órganos cosechables como los granos (Rodrigues Lima, 2005).

El objetivo del presente trabajo fue analizar la incidencia del daño mecánico en semillas de poroto sobre el desarrollo y el rendimiento de plantas normales y anormales.

\section{MATERIALES Y MÉTODOS}

Material biológico: Para el presente trabajo se utilizaron dos lotes de semillas de poroto (Phaseolus vulgaris $\mathrm{L}$.), pertenecientes a un cultivar blanco Paloma INTA y a uno negro Camilo INTA cosecha 2005, de primera multiplicación, provistos por la Estación Experimental INTA Cerrillos-SaltaArgentina.

Contenido de humedad: Fue evaluado gravimétricamente después de secar tres repeticiones de 100 semillas de cada cultivar a $105 \pm 3{ }^{\circ} \mathrm{C}$ por 24 horas (Brasil, 1992) obteniéndose un valor 12,3\%.

Tratamientos: Con el propósito de disponer de semillas con diferencias en su calidad, los lotes de cada cultivar de poroto previamente curados con captan a razón de $1 \mathrm{~g} / \mathrm{kg}$, y divididos en tres sublotes, fueron sometidos a daño mecánico dejándolos caer: 0,2 y 4 veces sobre un plato metálico desde una altura de dos metros (Dickson \& Boettger, 1976). Posteriormente cada fracción fue almacenada en cámara a $2-5^{\circ} \mathrm{C}$ hasta el momento de su utilización en los ensayos programados.

\section{DETERMINACIONES EN LABORATORIO}

\section{VIABILIDAD}

Ensayo de germinación: Se sembraron cinco repeticiones de 10 semillas entre toallas de papel humedecido. Los rollos se colocaron en bolsas de polietileno transparente y fueron llevados a cámara de germinación $\left(20-30{ }^{\circ} \mathrm{C} ; 8\right.$ hrs luz y 16 hrs. oscuridad). Al noveno día (ISTA, 1996) se clasificaron las plántulas en normales, anormales y semillas muertas según el Manual de Evaluación de Plántulas (ISTA, 1978), expresándose los resultados en porcentajes.

Categorización de anormalidades: Teniendo en cuenta que en poroto las plántulas anormales muestran distintos tipos de defectos e insuficiencias tanto a nivel del sistema radical como en el apical, se identificaron y clasificaron exomorfológicamente las anormalidades según categorías de acuerdo a las descripciones establecidas en el Manual de Evaluación de Plántulas (ISTA, 1978).

\section{VIGOR}

Ensayo de crecimiento: Utilizando las plántulas normales y anormales de cada cultivar y tratamiento de poroto obtenidas de cada test de germinación, se procedió a valorar pesos frescos y secos de parte aérea y de raíz (g). Con los respectivos datos se calculó el siguiente Índice de vigor (Ram et al., 1991):

$$
\mathrm{IV}=(\mathrm{PS} \times \mathrm{G})
$$

PS = peso seco total de plántula; $\mathrm{G}=$ porcentaje de germinación. 


\section{DETERMINACIONES EN CONDICIONES DE CAMPO}

Los ensayos se llevaron a cabo en San Salvador de Jujuy ( $65^{\circ} 17^{\prime} \mathrm{W}, 24^{\circ} 11^{\prime}$ S). Jujuy, Argentina, en el período 2006/2007.

Selección de plántulas anormales: La presencia de las diferentes categorías de anormalidades se expresó en porcentajes sobre el total de semillas utilizadas. Los mismos fueron ordenados según la frecuencia de su aparición. De este modo, para ambos cultivares de poroto se identificaron los mayores porcentajes de plántulas entre algunas que evidenciaban anormalidades en la parte aérea, mostrando hipocótilo y/o epicótilo cortos o ausentes.

Evaluación de etapas reproductivas: Las plántulas normales y anormales de seis días de edad seleccionadas de cada cultivar y tratamientos fueron transferidas - en condiciones de invernáculo-a macetas con sustrato tierra de monte: arena en proporción 2:1. Después de una semana, se llevaron a condiciones de campo incorporándolas a macetas de 7,5 L. de capacidad conteniendo el mismo sustrato, las que mantuvieron la humedad necesaria mediante aportes de riegos.

Para valorar las etapas del ciclo de vida se consignaron las fechas de inicio de las siguientes fases:

R6 $($ Floración $)=$ Se la consideró cuando el $50 \%$ de las plantas en estudio presentaban la primera flor abierta,

R7 (Formación de vainas $)=$ Fue determinada cuando el $50 \%$ de las plantas mostraron la primera vaina con la corola de la flor colgada o desprendida,

R9 $($ Maduración $)=$ Fue definida cuando el $50 \%$ de las plantas presentaban decoloración y secado de las vainas.

Valoración de componentes del rendimiento: Luego de registrar la etapa $\mathrm{R} 9$, y cuando las vainas presentaban un color verde amanzanado, se extrajeron las plantas completas, procediéndose a medir los siguientes componentes del rendimiento: números de vainas y de semillas/planta; número de semillas/vaina; longitud de vainas y peso de 1.000 semillas.

Con los valores obtenidos, se calculó el siguiente Índice de cosecha (Araujo et al., 1999):

$$
\text { IC = PS grano/PS aéreo }\left(\mathrm{g} \cdot \mathrm{g}^{-1}\right)
$$

$\mathrm{PS}=$ peso seco.
Diseño experimental y análisis estadístico: Para cada ensayo realizado se utilizó un diseño completamente aleatorizado con cinco repeticiones. El estudio estadístico de los resultados se llevó a cabo por el método de análisis de la varianza y las medias fueron estudiadas por el test de Tukey $(\mathrm{p} \leq 0,05)$ (Statistical Analysis System).

\section{RESULTADOS Y DISCUSIÓN}

Los resultados de las valoraciones de viabilidad y de vigor de semillas de poroto blanco y negro (Tabla 1) muestran que, frente al aumento del daño al que se sometieron a las semillas, si bien el porcentaje de germinación no disminuyó de modo significativo, se detecta la presencia de plántulas anormales, siendo ésta una de las características que identifican a semillas de baja calidad (Bewley, 1986). Asimismo, el Índice de vigor calculado para el cultivar Paloma INTA evidenció declinaciones significativas $(\mathrm{p} \leq 0,05)$ a nivel de plántulas anormales de todos los tratamientos respecto de las correspondientes plántulas normales, comportamiento que para el cultivar Camilo INTA sólo se expresó en el tratamiento cuatro caídas, resultados que expresan la ocurrencia de algún nivel de daño en la semilla (Begnami \& Cortelazzo, 1996; Trawatha et al., 1995), a la vez que ponen de manifiesto que poroto blanco es más sensible al deterioro que las semillas de color (Kannenberg \& Allard, 1964). Por otra parte, estas observaciones concuerdan con estudios previos que sostienen que el vigor de la semilla merma como consecuencia del envejecimiento (Delouche \& Baskin, 1973; Powell et al., 1984; Walters et al., 2001), lo que sugiere en ese proceso la existencia de alteraciones degenerativas en la semilla como consecuencia del daño mecánico (Tekrony, 2003).

El análisis de la ocurrencia de algunas de las fases reproductivas en ambos cultivares de poroto (Tabla 2) muestra que las plantas normales del cultivar Paloma INTA provenientes de los tratamientos con dos y cuatro caídas, adelantan las fases R6, R7 y R9; en tanto que las respectivas plantas anormales del cultivar Camilo INTA sólo lo hacen para las etapas R6 y R9. Estos resultados confirman observaciones que dan cuenta de variaciones en el cumplimiento de algunas fases reproductivas en plantas anormales de maní (Sullivan \& Perry, 1976). 
Tabla 1

Valoraciones de la viabilidad y el vigor de semillas de poroto blanco y de poroto negro sometidas a daño mecánico

\begin{tabular}{|c|c|c|c|c|c|}
\hline \multirow{2}{*}{ Cultivares } & \multirow{2}{*}{ Tratamientos } & \multirow{2}{*}{ Germinación (\%) } & \multicolumn{3}{|c|}{ Plántulas } \\
\hline & & & Tipos & $(\%)$ & Índice de vigor (PS x G) \\
\hline \multirow{6}{*}{$\begin{array}{l}\text { Paloma } \\
\text { INTA }\end{array}$} & \multirow{2}{*}{ Testigo } & \multirow{2}{*}{$58 \mathrm{a}$} & $\mathrm{N}$ & $58 \mathrm{a}$ & $14,26 \mathrm{a}$ \\
\hline & & & $\mathrm{AN}$ & $42 \mathrm{a}$ & $5,80 \mathrm{~b}$ \\
\hline & \multirow{2}{*}{2 caídas } & \multirow{2}{*}{$66 \mathrm{a}$} & $\mathrm{N}$ & $66 a$ & $16,50 \mathrm{a}$ \\
\hline & & & $\mathrm{AN}$ & $34 \mathrm{a}$ & $7,92 b$ \\
\hline & \multirow{2}{*}{4 caídas } & \multirow{2}{*}{$62 \mathrm{a}$} & $\mathrm{N}$ & $62 \mathrm{a}$ & $14,26 \mathrm{a}$ \\
\hline & & & AN & $38 \mathrm{a}$ & $6,32 b$ \\
\hline \multirow{6}{*}{$\begin{array}{l}\text { Camilo } \\
\text { INTA }\end{array}$} & \multirow{2}{*}{ Testigo } & \multirow{2}{*}{$56 a$} & $\mathrm{~N}$ & $56 a$ & $2,80 \mathrm{a}$ \\
\hline & & & AN & $18 \mathrm{a}$ & $2,60 \mathrm{a}$ \\
\hline & \multirow{2}{*}{2 caídas } & \multirow{2}{*}{$44 \mathrm{a}$} & $\mathrm{N}$ & $44 \mathrm{a}$ & $2,90 \mathrm{a}$ \\
\hline & & & $\mathrm{AN}$ & $32 \mathrm{a}$ & $2,34 \mathrm{a}$ \\
\hline & \multirow{2}{*}{4 caídas } & \multirow{2}{*}{$40 \mathrm{a}$} & $\mathrm{N}$ & $40 \mathrm{a}$ & $2,80 \mathrm{a}$ \\
\hline & & & $\mathrm{AN}$ & $30 a$ & $1,33 b$ \\
\hline
\end{tabular}

$\mathrm{N}=$ plántula normal; $\mathrm{AN}=$ plántula anormal.

Letras iguales indican diferencias no significativas $(\mathrm{p} \leq 0,05)$ según Tukey.

Tabla 2

Determinaciones de la presencia de tres fases reproductivas en plantas normales y anormales de los dos cultivares de poroto en estudio

\begin{tabular}{|c|c|c|c|c|c|}
\hline \multirow{2}{*}{ Cultivares } & \multirow{2}{*}{ Tratamientos } & \multirow{2}{*}{ Tipos de plántulas } & \multicolumn{3}{|c|}{ Días desde emergencia hasta alcanzar: } \\
\hline & & & R6 & $\mathbf{R 7}$ & R9 \\
\hline \multirow{6}{*}{$\begin{array}{l}\text { Paloma } \\
\text { INTA }\end{array}$} & \multirow{2}{*}{ Testigo } & $\mathrm{N}$ & 41 & 44 & 97 \\
\hline & & $\mathrm{AN}$ & 41 & 50 & 97 \\
\hline & \multirow{2}{*}{2 caídas } & $\mathrm{N}$ & 35 & 41 & 78 \\
\hline & & AN & 35 & 41 & 97 \\
\hline & \multirow{2}{*}{4 caídas } & $\mathrm{N}$ & 35 & 41 & 83 \\
\hline & & AN & 35 & 41 & 91 \\
\hline \multirow{6}{*}{$\begin{array}{l}\text { Camilo } \\
\text { INTA }\end{array}$} & \multirow{2}{*}{ Testigo } & $\mathrm{N}$ & 41 & 45 & 103 \\
\hline & & $\mathrm{AN}$ & 44 & 57 & 103 \\
\hline & \multirow{2}{*}{2 caídas } & $\mathrm{N}$ & 50 & 57 & 103 \\
\hline & & AN & 35 & 50 & 97 \\
\hline & \multirow{2}{*}{4 caídas } & $\mathrm{N}$ & 41 & 44 & 97 \\
\hline & & $\mathrm{AN}$ & 35 & 41 & 83 \\
\hline
\end{tabular}

$\mathrm{N}$ = plántula normal; $\mathrm{AN}$ = plántula anormal. 
Respecto de los componentes del rendimiento valorados en poroto blanco (Tabla 3 ) se determinó que en las plantas anormales de los tratamientos dos y cuatro caídas declinaron las cantidades de vainas y de semillas/planta, siendo únicamente significativas $(p \leq 0,05)$ esas mermas en el primer tratamiento, mientras que en poroto negro (Tabla 4) las correspondientes al lote control aumentaron entre el $19-37 \%$ y en menor proporción para el tratamiento de dos caídas, componentes que por otra parte alcanzan variaciones similares debido a su directa relación con la productividad final del cultivo (Prijic et al., 1991).

Estos resultados demuestran que las mermas en los componentes del rendimiento descritas por ISTA (1985) para plantas anormales se manifiestan de modo particular según la especie y el cultivar estudiado.

En cuanto al número de semillas/vaina y al peso de 1.000 semillas, ambos cultivares de poroto no evidenciaron diferencias significativas cuando se comparan plantas normales y anormales.

Tabla 3

Evaluaciones de componentes del rendimiento desde plantas normales y anormales de poroto blanco

\begin{tabular}{|c|c|c|c|c|c|c|c|c|}
\hline \multirow[b]{2}{*}{ Cultivar } & \multirow[b]{2}{*}{ Tratamientos } & \multirow{2}{*}{$\begin{array}{c}\text { Tipos de } \\
\text { plántulas }\end{array}$} & \multicolumn{5}{|c|}{ Componentes del rendimiento } & \multirow{2}{*}{$\begin{array}{c}\text { Índice de } \\
\text { cosecha } \\
\left({\left.\mathrm{g} \times \mathrm{g}^{-1}\right)}^{-1}\right.\end{array}$} \\
\hline & & & $\begin{array}{c}\mathrm{N}^{\circ} \mathrm{de} \\
\text { vainas x pl. }\end{array}$ & $\begin{array}{c}\text { Long. de } \\
\text { vainas }(\mathrm{cm})\end{array}$ & $\begin{array}{c}\mathrm{N}^{\mathbf{o}} \text { de semillas } \\
\mathrm{x} \text { vaina }\end{array}$ & $\begin{array}{c}\mathrm{N}^{0} \text { de semillas } \\
\quad \mathrm{x} \mathrm{pl.}\end{array}$ & $\begin{array}{l}\text { Peso de } \\
1.000 \text { s. }(g)\end{array}$ & \\
\hline \multirow{6}{*}{$\begin{array}{l}\text { Paloma } \\
\text { INTA }\end{array}$} & \multirow{2}{*}{ Testigo } & $\mathrm{N}$ & $16,6 \mathrm{a}$ & $11,62 \mathrm{a}$ & $3,07 \mathrm{a}$ & $51 \mathrm{a}$ & $613,9 \mathrm{a}$ & $1,40 \mathrm{a}$ \\
\hline & & AN & $15 \mathrm{a}$ & $10,42 \mathrm{a}$ & $2,87 \mathrm{a}$ & $43 \mathrm{a}$ & $518,1 \mathrm{a}$ & $2,29 b$ \\
\hline & \multirow{2}{*}{2 caídas } & $\mathrm{N}$ & $21 \mathrm{a}$ & $11,97 \mathrm{a}$ & $2,88 \mathrm{a}$ & $60 \mathrm{a}$ & $647,7 \mathrm{a}$ & $1,48 \mathrm{a}$ \\
\hline & & AN & $12,3 \mathrm{~b}$ & $10,79 \mathrm{a}$ & $2,68 \mathrm{a}$ & $32 b$ & $562,5 \mathrm{a}$ & $2,51 \mathrm{~b}$ \\
\hline & \multirow{2}{*}{4 caídas } & $\mathrm{N}$ & $23,6 \mathrm{a}$ & $12,24 \mathrm{a}$ & $3,34 \mathrm{a}$ & $79 \mathrm{a}$ & $613,2 \mathrm{a}$ & $1,33 \mathrm{a}$ \\
\hline & & AN & $20,3 \mathrm{a}$ & $11,60 \mathrm{a}$ & $3,11 \mathrm{a}$ & $63 \mathrm{a}$ & $557,7 \mathrm{a}$ & $1,47 \mathrm{a}$ \\
\hline
\end{tabular}

$\mathrm{N}$ = plántula normal; $\mathrm{AN}$ = plántula anormal

Letras iguales indican diferencias no significativas $(\mathrm{p} \leq 0,05)$ según Tukey.

Tabla 4

Evaluaciones de componentes del rendimiento desde plantas normales y anormales de poroto negro

\begin{tabular}{|c|c|c|c|c|c|c|c|c|}
\hline \multirow[b]{2}{*}{ Cultivar } & \multirow[b]{2}{*}{ Tratamientos } & \multirow{2}{*}{$\begin{array}{l}\text { Tipos de } \\
\text { plántulas }\end{array}$} & \multicolumn{5}{|c|}{ Componentes del rendimiento } & \multirow{2}{*}{$\begin{array}{c}\text { Índice de } \\
\text { cosecha } \\
\left(\mathrm{g} \times \mathrm{g}^{-1}\right)\end{array}$} \\
\hline & & & $\begin{array}{c}\mathrm{N}^{0} \text { de } \\
\text { vainas x pl. }\end{array}$ & $\begin{array}{c}\text { Long. de } \\
\text { vainas }(\mathrm{cm})\end{array}$ & $\begin{array}{c}\mathrm{N}^{\mathbf{o}} \text { de semillas } \\
\text { x vaina }\end{array}$ & $\begin{array}{c}\mathrm{N}^{\circ} \text { de semillas } \\
\quad \mathrm{x} \mathrm{pl.}\end{array}$ & $\begin{array}{c}\text { Peso de } \\
1.000 \text { s. (g) }\end{array}$ & \\
\hline \multirow{6}{*}{$\begin{array}{l}\text { Paloma } \\
\text { INTA }\end{array}$} & \multirow{2}{*}{ Testigo } & $\mathrm{N}$ & $47 \mathrm{a}$ & $9.45 \mathrm{a}$ & $4,63 a$ & $213,3 \mathrm{a}$ & $326,4 a$ & $1,48 \mathrm{a}$ \\
\hline & & AN & $56,3 \mathrm{a}$ & $9,13 \mathrm{a}$ & $5,28 \mathrm{a}$ & $292,6 a$ & $285,7 \mathrm{a}$ & $1,51 \mathrm{a}$ \\
\hline & \multirow{2}{*}{2 caídas } & $\mathrm{N}$ & $29 \mathrm{a}$ & $7,43 \mathrm{a}$ & $4,41 \mathrm{a}$ & $128 \mathrm{a}$ & $233,2 \mathrm{a}$ & $2,16 a$ \\
\hline & & AN & $30,6 \mathrm{a}$ & $9,05 \mathrm{a}$ & $4,68 \mathrm{a}$ & $147,3 b$ & $278,4 a$ & $2,12 \mathrm{a}$ \\
\hline & \multirow{2}{*}{4 caídas } & $\mathrm{N}$ & $39,3 \mathrm{a}$ & $8,79 a$ & $5,56 \mathrm{a}$ & $214 \mathrm{a}$ & $270 \mathrm{a}$ & $1,38 \mathrm{a}$ \\
\hline & & AN & $41,3 a$ & $7,80 \mathrm{a}$ & $4,98 \mathrm{a}$ & $210 \mathrm{a}$ & $229,6 \mathrm{a}$ & $1,56 a$ \\
\hline
\end{tabular}

$\mathrm{N}$ = plántula normal; $\mathrm{AN}=$ plántula anormal.

Letras iguales indican diferencias no significativas ( $\mathrm{p} \leq 0,05)$ según Tukey. 
Por otra parte, el Índice de cosecha calculado para poroto blanco y negro, considerando que el mismo expresa la proporción de materia orgánica acumulada en granos y que se asocia a la eficiencia del vegetal en producir biomasa (Araujo et al., 1999; Rodrigues Lima, 2005), en poroto blanco las plantas anormales de testigo y dos caídas mostraron incrementos significativos $(\mathrm{p} \leq 0,05)$ que alcanzaron en promedio un $65 \%$, mientras que en poroto negro sólo para el tratamiento con cuatro caídas hubo un aumento del $13 \%$ en comparación con los valores obtenidos desde plantas normales. Estos resultados indicarían que las plantas anormales alcanzaron durante la estación de crecimiento algún grado de recuperación, lo que contribuyó a mejorar la eficiencia de partición de fotoasimilados hacia los órganos cosechables, aspecto que por otro lado concuerda con las observaciones realizadas en maní (Lovey, 1999) y en soja (Prijic et al., 1991) en oportunidad de comparar el comportamiento demostrado por las plantas normales frente a las anormales.

\section{LITERATURA CITADA}

ARAUJO, A.; RODRIGUES LIMA, E.; DE OLIVEIRA, A.; DIAS, P.; TEIXEIRA, M. 1999. Índice de coheita de nitrogenio e de fosforo de cultivares de feijoeiro. VI Renafe. Reniao Nacional de Pesquisa de Feijao. Goiania. Goias. Brasil.

BEGNAMI, C.; CORTELAZZO, A. 1996. Cellular alterations during accelerated ageing of French bean seeds. Seed Sci \& Technol. 24: 295-303.

BEWLEY, D. 1986. Physiology of seed deterioration. Edit Mc Donald, M.B. \& Nelson, C.J. Madison. Wisconsin. USA, cap. 2, pp. 27-43.

BRASIL. 1992. Ministerio da Agricultura. Secretaria Nacional da Defesa Agropecuaria. Regras para Análise de sementes. Rules for Seed Testing. Brasilia. $188 \mathrm{sp.}$

COLETE, J.; VIEIRA, R.; DUTRA, A. 2004. Electrical conductivity and soybean seedling emergence. Seed Agric. Piracicaba. Braz. V 61,n 4: 386-391.

DELOUCHE, J.; BASKIN, C. 1973. Accelerated aging techniques for predicting the relative storability of seed lots. Seed Sci \& Technol. 1: 427-452.

DICKSON, M.; BOETTGER, M. 1976. Factors associated with resistance to mechanical damage in snap bean (Phaseolus vulgaris L.) . J. Amer. Soc. Hort. Sci 101 (5): 541-544.

FINCH-SAVAGE, W. 1995. Influence of seed quality on crop establishment, growth and yield. In Seed Quality: basic mechanisms and Agricultural implication (ed. A.S. Basra), pp. 361-384. Food Products press. NY.

ISTA. 1978. Manual de Evaluación de Plántulas. Instituto Nacional de Semillas y Plantas de Vivero. Madrid.

\section{CONCLUSIONES}

Las observaciones presentadas permiten sostener que:

- De los dos cultivares de poroto en estudio, Paloma INTA es el más sensible al daño mecánico,

- Si bien las plántulas anormales de todos los tratamientos de poroto blanco y las de cuatro caídas de poroto negro se mostraron menos vigorosas que las normales, y en ambos tipos de plántulas se determinaron variaciones para la ocurrencia de las fases reproductivas R6, R7 y R9, los resultados obtenidos sobre los componentes del rendimiento analizados indican que en las plántulas anormales se asociaron por efectos compensatorios mejoras en la partición de la biomasa producida hacia los órganos cosechables, lo que les permitió mantener en poroto negro y superar en el blanco los valores del Índice de cosecha calculados para las plantas normales.

ISTA. 1985. International Rules for Seed testing. Seed Sci \& Technol, 13: 300-520.

ISTA. 1996. Internacional Rules for Seed Testing. Seed Sci \& Technol., 21, supplement.

KANNENBERG, L.; ALLARD, R. 1964. An association between pigment and lignin formation in the seed coats of the lima bean. Crop Sci, 1 (2): 621-622.

LOVEY, R. 1999. Morfofisiología de plántulas anormales de maní (Arachis hypogaea L.), análisis histológico y evaluación del crecimiento y desarrollo. Seminario II. Maestría en Tecnología de Semillas. Univ. Nac. de Córdoba. Argentina, pp. 22.

POWELL, A.; MATTHEWS, S.; OLIVEIRA, M. 1984. Seed quality in grain legumes. Advances in applied Biology, 10: 217-285.

PRIJIC, L.; JOVANOVIC, M.; POPOVIC, R. 1991. Effect of abnormal seedling on major characters and grain yield in soybean. Seed Sci \& Technol., 19: 67-71.

RAM, C.; SINGH, O.; KHARD, R.; KUMARI, P., YADAVA, T. 1991. Seedling vigour in pigeonpea. Seed Sci \& Technol. 19: 627-631.

RODRIGUES LIMA, E.; SILVA SANTIAGO, A.; ARAUJO, A.; TEIXEIRA, M. 2005. Effects of the size of sown seed on growth and yield of common bean cultivars of different seed sizes. Braz. J. Plant Physiol. 17 (3): 273-281.

SULLIVAN, G.; PERRY, A. 1976. Comparative field performance of plants developing from normal and abnormal seedling of peanuts. Peanut Science, 3: 29-31.

TEKRONY, D. 2003. Precision is an essential component in seed vigour testing. Seed Sci \& Technol., 31: 435-447. 
TRAWATHA, S.; TEKRONY, D.; HIDEBRAND, D. 1995. Relationship of soybean seed quality to fatty acid and C6-aldehide levels during storage. Crop Sci, 35: 14151422.
WALTERS, C.; PAMMENTER, N.; BERJAK, P.; CRANE,

J. 2001. Desiccation damage accelerated ageing and respiration in desiccation tolerant and sensitive seeds. Seed Sci Research, 11, 135-148. 